\title{
Chikungunya - an emerging infection in Bangladesh
}

\author{
MN Islam ${ }^{1}$
}

\section{Introduction}

Chikungunya fever is a mosquito-borne illness of humans caused by the chikungunya virus, a type of alphavirus. Aedesaegypti and Aedesalbopictus mosquitoes are the main vectors of chikungunya in Asia and the Indian Ocean islands. The virus was first reported in 1952 in Tanzania. Since then it has been attributed to many outbreaks in a number of countries. The virus is geographically distributed in Africa, Southeast Asia and India. Sporadic cases are regularly reported from different countries in the affected regions . During December 2008, an investigation team from the Institute of Epidemiology, Disease Control and Research (IEDCR) and International Centre for Diarrhoeal Disease Research, Bangladesh (ICDDR,B) investigated the first outbreak of chikungunya fever in the Rajshahi and Chapianawabganj districts of Bangladesh, which was in fact the third outbreak in the whole of Bangladesh. We identified six cases of chikungunya fever in the last year.

\section{Discussion}

Chikungunya fever (CF) is a viral illness caused by an arbovirus transmitted by the Aedes mosquitoes. The disease was documented first time in the form of an outbreak in Tanzania. The name is derived from the 'makonde'dialect which means 'that which bends up', indicating the physical appearance of a patient with severe clinical features. First described in Africa in 1952 and then identified in Asia, it was responsible for widespread outbreaks on these two continents from the 1960s to the 1980 its global emergence really started in 2004.A major outbreak first spread, to most islands in the Indian Ocean in 2005-2006. CHIKV spread progressively toward Asia, affecting India since 2006 and successively most countries of the Region. The first identified Chikungunya outbreak occurred in Bangladesh in 2008 in the northwest area of the country. In 2011 it was reported in Dohar, Dhakadistrict.In 2017 it again comes back as a outbreak in Dhaka. The fever locally also named as 'Langrajor.

The most significant manifestation of chikungunya fever is the severe joint pain occurring in virtually every clinical case. The arthralgia is most commonly symmetrical and peripheral, being noted in the small joints of the hands and other large joints. The joints exhibit extreme tenderness and swelling with patients frequently reporting incapacitating pain that lasts for weeks to months. Most infections completely resolve within weeks or months but there have been documented cases of chikungunya fever-induced arthralgia persisting for several years.patients had residual joint pain for two to three months after recovering from the initial infection.

The symptoms of infection are quite similar to those caused by many other infectious agents in the endemic areas. One particular difficulty in identifying infection is its overlapping distribution with dengue virus. It has been postulated that many cases of dengue virus infection are misdiagnosed and in practice the incidence of chikungunya infection is much higher than reported Diagnosis of chikungunya is based on two cardinal signs in the acute phase; fever and

${ }^{1}$ Assistant Professor, Department of Medicine, Rajshahi Medical College, Rajshahi- 6204. 
arthralgia has a specificity of $99.6 \%$ and positive predictive value of $84.6 \%$. However, as the clinical manifestations of chikungunya fever resemble those of dengue and other fevers caused by arthropod-borne viruses, confirmation of chikungunya fever should be based on: isolation of the virus, molecular methods, detection of IgM antibody, and demonstration of a rising titer of the IgGantibody . In Bangladesh, only detection of IgMAb is so far possible in Dhaka city. We confirmed our diagnosis by detecting IgMAb against chikungunya virus. Now it is confirmed by RT PCR which is available in IEDCR,Bangladesh. A specific antiviral agent or vaccine against chikungunya was not available till now. Treatment is supportive, involving rest, proper diet, movement and mild exercise. Combinations with mild pain relief medication, such as naproxen, ibuprofen, acetaminophen or paracetamol, may relieve the fever and aches. Re-evaluation and closer monitoring are advised in chronic ailments. Chikungunya virus infection provides immunity against the disease .

\section{Conclusion:}

Chikungunya fever is not uncommon in Bangladesh. Most of the cases remain undiagnosed or misdiagnosed due to lack of awareness and diagnostic facilities, the selflimiting nature of the disease and, most importantly, the prevalence of another arthropodborne disease, dengue fever, in Bangladesh. Clinically, it can be distinguished from dengue fever but laboratory diagnosis is a prerequisite for confirmation.

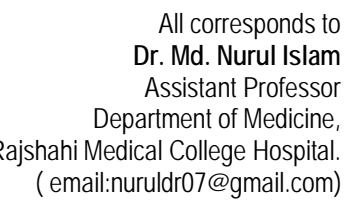

